\title{
Caregivers
}

\section{OP 013 CLARITY THROUGH THE KALEIDOSCOPE: GAINING CONSENSUS ON THE MAIN CAUSES OF CARER BURDEN FROM PROFESSIONAL AND CARER PERSPECTIVES}

Katherine Knighting, ${ }^{1}$ Barbara Jack, ${ }^{1}$ Brenda Roe, ${ }^{1}$ Mary O'Brien, ${ }^{1}$ Mike Nolan, ${ }^{2}$ Mari Lloyd-Williams, ${ }^{3}$ Kirsty Pine, ${ }^{4}$ Rob Gandy ${ }^{1} .{ }^{1}$ Edge Hill University, Ormskirk, UK; ${ }^{2}$ Sheffield University, Sheffield, UK; ${ }^{3}$ University of Liverpool, Liverpool, UK; ${ }^{4}$ Liverpool Clinical Commissioning Group, Liverpool, UK

10.1136/bmjspcare-2014-000654.13

Background Family caregivers of patients with cancer and other advanced progressive illnesses, especially during the final year of life, can incur increasing burden, affecting their health, ability to care and likelihood of hospital admission for the patient. It is essential carers' needs are assessed to alert health and social care professionals to their increasing level of burden and trigger additional support. This multi-phase study developed and piloted a Carers' Alert Thermometer (CAT), for use by the generalist healthcare workforce, to alert staff to increasing carer burden and provide guidance on appropriate interventions.

Aim of the study To gain consensus on the most important burdens raised by carers for inclusion in the CAT (Phase 1) subsequently rated and ranked by professionals and carers (Phases 2 and 3).

Methods Phase 2: A two-round Delphi survey was conducted with carers, health and social care professionals and organisations supporting carers. Round 1 involved 44 burdens in eight main themes. Round 2 contained 29 burdens which had been rated as 'extremely important' or where a high level of disagreement between carers and professionals existed.

Phase 3: A virtual panel, comprised of carers together with professionals from national and regional organisations with strategic roles in end-of-life care and carer support, commented on the Delphi findings ranking their top 10 burdens for inclusion in the CAT.

Results There was a high level of agreement between the professionals and carers on the main burdens for inclusion in the CAT. Understanding the current caring situation and supporting carer's health and well-being were the main priorities. Interestingly, end-of-life planning was ranked lowest by both groups.

Conclusions Despite the complex needs of carers clear consensus on the main burdens exists which can be utilised in an alert tool to identify increasing burden and guide appropriate targeting of support and resources. 